diseases of the joints, such as ankylosing spondylitis, is omitted.

Chapter 10 consists of a series of paragraphs on different topics, otherwise unmentioned, which are arranged alphabetically rather than in a related sequence. One important point made under the heading of paternity is awareness that non-paternity is higher in a child conceived before marriage, and that care is needed therefore before giving the poor prognosis for an autosomal recessive disorder found in a first born child.

There are quite a number of misprints including two on p 31 and the index is not always accurate. Amniocentesis does not appear on p 68 but there are important sections on $p$ 69. No references are given by design in the text but there are a number of monographs or reviews given under suggestions for further reading, together with the author's useful comments on their value.

Although the intention of this book is commendable, in that of starting from the basic set of components, it has not succeeded. Whether this analytical approach would appeal to many medical students is open to question, but before it could be recommended, considerable rewriting would be required. Furthermore, there are other volumes of similar size which meet the needs of the medical student. Whether it would be more useful to science graduates with some knowledge of genetics is also uncertain because of their unfamiliarity with many of the medical diagnoses mentioned but not described.

A W Johnston

\section{Genetics and Cancer in Man}

By $R$ N Schimke. (Pp viii +108 ; Figures + Tables. $£ 7 \cdot 00$.) Edinburgh, London, New York: Churchill Livingstone. 1978.

Malignancy is a major cause of human morbidity and mortality. The most common forms of neoplasia occur in later adult life, and this makes both prospective genetic studies and the retrospective collection of adequate family data more difficult. These, added to the many other difficulties of investigation of the inheritance of human disorders, have tended to turn many away from the study of cancer genetics in man. Yet there is increasing evidence that among those patients with the common neoplasms there is a group of families in whom cancer predisposition behaves for practical purposes as a simply inherited trait. For example, among the wide and apparently random distribution of carcinoma of the colon in the general population, it is seen regularly as a development in the hereditary polyposis syndromes where it behaves like a straightforward dominant, although the bulk of colonic cancer cases may not be genetic at all. But even in those families in which the genetic risk of malignancy is high, there remain some disturbing features. Monozygotic twin concordance is frequently far less than expected, even when allowance is made for differences in expression and lack of penetrance.

The volume by Professor Schimke on genetics and cancer in man is very welcome. It is part of the series published by Churchill Livingstone under the general editorship of Professor Alan Emery designed to provide trainee and practising doctors with easily accessible and authoritative information about genetic aspects of their specialities. There are nine chapters. The first is introductory, setting out the difficulties of cancer genetics, the origin of heritable tumours in man, pitfalls in the analysis of sitespecific neoplasia, malignancy in recessive disorders, the association with malignancy of the HLA system and cytogenetics, and the clonal origin of tumour cells.

Chapter 2 concerns embryonal tumours, uncommon except for haemangiomas, lymphangiomas, and various types of pigmented naevi. It is tacitly assumed that they originate in maldeveloped fetal tissue, although they may not become clinically evident until childhood, the incidence declining rapidly with advancing age, so that most develop before the age of 5 . The usually solid embryonal tumours contain histologically mixed types of tissue or are sarcomatous and usually are not considered heritable. But nephroblastoma, retinoblastoma, neuroblastoma, hepatoblastoma, the sarcomas, and teratoma show quite distinct hereditary predispositions. In the first two approximately one-third show clear evidence of inheritance, the remainder a much lower familial tendency.

The next chapter deals with the hamartomas, solid or cystic tumours formed by excessive aggregation of either single or multiple tissue elements, and the hamartoma syndromes where different organs throughout the body contain these conglomerates. Here neurofibromatosis, tuberous sclerosis, von Hippel-Lindau syndrome, and the basal cell naevus syndrome are discussed, all of which show clear evidence of different forms of Mendelian inheritance, while in the syndromes too, for example the Gardner syndrome, the Peutz-Jegher syndrome, the Turcot syndrome, the evidence for Mendelian inheritance is strong.

In the next chapter on cancers of the skin, the summary table of skin abnormalities as indicators of potential non-cutaneous malignancy is particularly useful. So too in chapter 5 on endocrine gland 
neoplasias is the table of screening studies helpful in patients at risk for type I multiple endocrine neoplasia (Werner syndrome), while the intriguing concept of the APUD cells, suggesting homogeneity of origin of the cells that migrate into the primitive foregut and presumably develop into the endocrine system, is shown to have stimulated considerable activity.

Chapters follow on the gastrointestinal cancers, which provide some of the most difficult cases for counselling, and the tumours of the reproductive system and leukaemia. Prostate and testicular tumours are rare, but of cancers of the ovary there are numerous reports of familial occurrence, the trait apparently being transmitted by a male. Tumours in intersex patients are dealt with briefly, and prophylactic removal of intra-abdominal gonads or gonadal remnants shortly after expected puberty is recommended.

This book is useful, both for the remarkably succinct account of the cancers in which an inherited component is identifiable, and also for its clear exposition of what is not known. Many a voyage of discovery has foundered on the hidden rocks of tumour investigation, the complications of environmental and genetic predisposition interaction. Yet the survivors have given us remarkable evidence of the strength of the genetic involvement in a number of neoplastic states.

This modest little book, and the evidence so clearly marshalled within it, will serve as an excellent first guide to genetic counselling in relevant situations, and as a baseline for the accumulation of additional information.

\section{F ROBERTS}

\section{Chemical Mutagens. Principles and Methods for their Detection}

Vol 5. Edited by A Hollaender and F J de Serres. $(\mathrm{Pp} \mathrm{xv}+348$; Figures + Tables. $\$ 18 \cdot 58$. $)$ New

York and London: Plenum Press. 1978.

The explosive development during the past decade of short term tests for the detection of mutagens and carcinogens has been reflected in previous volumes in this series. Too little thought, however, has been given to the way in which information generated by these tests should be used and to the problem of relating it to possible effects in man. The present volume shows a clear emphasis on effects in laboratory mammals (as distinct from micro-organisms or mammalian cells) and this must be welcomed as $\stackrel{\mathbb{D}}{=}$ trend in the right direction.

The clinician interacts with this type of work most often when he suspects that a patient may have been $\frac{-}{-}$ exposed to an occupational or environmentalo carcinogen. There are three chapters that are particularly relevant to this. The first, by Legator, 잉 Truong, and Connor, describes techniques for the detection of mutagens in body fluids, both of mice and $\vec{o}$ men. Mutagenic activity has, for example, beendetected in the urine of workers exposed to high $\vec{\rho}$ levels of epichlorhydrin. The alkylation of histidine residues in haemoglobin is another method that canô be used to detect occupational exposure to alky- $\vec{v}$ lating agents.

A second chapter by Wyrobek and Bruce discusses the use of sperm morphology abnormalitieso as an indicator of exposure to agents capable of ${ }_{-}^{\supset}$ inducing gene mutations. There is little doubt that ${ }_{T}$ mutagens can give rise to sperm shape abnormalities ${ }^{\mathbb{D}}$ in the mouse, but the usefulness of the response is limited by the fact that abnormalities can also be caused by physiological disturbance so that $a-\overrightarrow{0}$ positive effect must always be considered in the lighto of information from a variety of other systems. In. man there is considerable person-to-person variability in the proportion of morphologically abnormal sperm. Nevertheless, the approach has been used; for example, it has been shown that the proportiono of abnormal sperm correlates well with the numberฉ of cigarettes smoked per day.

Perhaps the most interesting chapter is that by Miller which reviews the discovery of human? teratogens, carcinogens, and mutagens and points: some lessons for the future. By far the most impor tant initiators of discovery have been alert practi-ซo tioners. Despite the development of short termo tests and record linkage, it is still the practitioner who has a vital role in uncovering effects in man.o Epidemiology may be useful in confirming suspicions but it has rarely been useful in formulating them.을 Of course, many of the clinician's suspicions will be unfounded, and some may be untestable through음 lack of an adequate number of exposed persons. $N$ What would be useful, however, would be a place S $^{\text {I }}$ of referral where practitioners could send their 0 suspicions for evaluation by experts. This would N need to be in the hands of the medical professiono but with full access to advisers in the areas of environmental mutagenesis, cancer research, and $\frac{C}{\overparen{C}}$ epidemiology.

B A BRIDGEST 